\title{
Propagation of two-dimensional vibroacoustic disturbances in a rarefied gas
}

\author{
A. Manela (1* \\ Faculty of Aerospace Engineering, Technion-Israel Institute of Technology, Haifa 32000, Israel \\ Y. Ben-Ami 10 \\ Wolfson Centre for Mathematical Biology, Mathematical Institute, University of Oxford, \\ Woodstock Road, Oxford OX2 6GG, England, United Kingdom
}

(Received 10 June 2021; accepted 13 August 2021; published 1 September 2021)

\begin{abstract}
We study the effect of gas rarefaction on the propagation of vibroacoustic sound in a two-dimensional setup of a gas confined by a planar oscillating wall. Focusing on small-amplitude harmonic excitations imposed to only part of the surface, the problem is analyzed in the entire range of gas rarefaction rates, governed by the ratio between the gas mean free path and wavelength of a prescribed wall signal. Analytical solutions are obtained in the free-molecular and continuum limits, consisting of the gas response to a delta function and other locally confined wall actuations. The analysis is supplemented by direct simulation Monte Carlo calculations in the intermediate range of gas rarefaction rates. Remarkably, distinct differences are observed between sound propagation characteristics in the two limits. At continuum-limit conditions, the acoustic signal propagates isotropically in a monopole-type pattern, and decays inversely to the square root distance from the source. In contrast, the signal in the collisionless flow regime is exponentially decaying away from the source and follows a "nearly dipole-type" directivity field, where the acoustic pressure vanishes in the direction normal to the source axis of motion. The differences in results, stemming from the fundamentally distinct continuum and ballistic flow models, are discussed and rationalized in terms of the analytical limit-case descriptions.
\end{abstract}

DOI: 10.1103/PhysRevFluids.6.093401

\section{INTRODUCTION}

The generation and propagation of acoustic waves in rarefied gases have been investigated in a considerable number of works, starting in the middle of the 20th century and continuing to current research [1-9]. Initially motivated by engineering low-pressure applications and fundamental interest, it has been observed that the departure from continuum in a given acoustic setup becomes relevant wherever the characteristic lengthscale, or timescale, of the configuration involved becomes of the order of its kinetic microscopic counterparts, namely, the mean free path or time, respectively. In vibroacoustic applications, such scenarios are common in micromechanical oscillators, where short lengthscales and high frequencies take place [10-12].

Initially focusing on a uniformly vibrating planar setup (see Refs. [2,5-8,13] and papers cited therein), a major part of existing studies has analyzed the attenuating effect of gas rarefaction on sound propagation, quantitating an increase in the signal decay rate with departure from continuum conditions. A parallel set of studies has then examined the thermoacoustic field generated by temperature changes [14-19] or heat flux variations [20-22] of a surface, suggesting it as a means for vibroacoustic sound cancellation. Following investigations have analyzed vibroacoustic and

*amanela@technion.ac.il 
thermoacoustic sound generation at nonplanar geometries, by considering the canonical setups of a pulsating cylinder [23,24] and a pulsating sphere [25].

Invariably, the above works examine acoustic wave propagation at one-dimensional configurations. These include both planar- and non-planar-wall investigations, where axisymmetric flow conditions have been assumed in the latter [23-25]. While the imposition of unidirectional sound propagation significantly simplifies the analysis, it appears desirable to extend existing works and investigate the effect of gas rarefaction on sound transmission at more complex and realistic source configurations. In an effort in this direction, Wu [26] has examined the propagation of acoustic waves in a rarefied gas confined in a two-dimensional cavity. Uniform harmonic oscillations of one of the cavity walls were considered as the system source of sound, and the analysis combined numerical solution of the linearized Boltzmann equation with analytic investigation of the collisionless (high oscillation frequency) limit. In other works, Sader and coworkers [19,27] have considered the acoustic field of an oscillating rigid sphere, taking into account the prevailing nonaxisymmetric conditions and applying the Bhatnagar-Gross-Krook model of the Boltzmann equation for the analysis.

Observing the few number of works studying sound propagation at non-one-dimensional setups, the objective of the present paper is to analyze the particular effect of gas rarefaction on the propagation of two-dimensional vibroacoustic sound generated by localized boundary motion. To this end, we consider a semi-infinite expanse of a gas confined by an infinite planar wall undergoing nonuniform time-depending normal vibrations. This may be assumed as a simple model for characterizing sound transmission by a locally vibrating panel (speaker) at noncontinuum conditions. The problem is formulated for an arbitrary small-amplitude profile of surface vibration, and the effect of system two-dimensionality is emphasized by focusing on particularly "localized" signal distributions, as opposed to uniform planar motion considered in previous one-dimensional investigations. The problem is studied in the entire range of gas rarefaction rates, governed by the ratio between the gas molecular mean free path and the characteristic wavelength of imposed vibrations. Remarkably, fundamentally distinct acoustic flow fields are observed in the highly rarefied (free-molecular) and continuum limit regimes, in terms of both signal decay rate and directivity pattern. These differences are rationalized based on respective analytical solutions derived in the two limits. The results are supplemented by direct simulation Monte Carlo (DSMC) calculations, used to bridge the gap between the two limits and obtain the acoustic-field topology at intermediate rarefaction rates.

In the next section, the acoustic problem is stated. The analyses of the free-molecular and continuum limits are given in Secs. III and IV, respectively, and the direct simulation Monte Carlo algorithm applied is discussed in Sec. V. The results, shedding light on the fundamental effects of gas rarefaction on two-dimensional sound propagation, are presented in Sec. VI, and our conclusions are given in Sec. VII. Some technical details are relegated to the Appendices.

\section{STATEMENT OF THE PROBLEM}

A schematic of the problem is shown in Fig. 1. Consider a semi-infinite expanse of a monatomic ideal gas located at $y^{*}>0$ and confined by an infinite solid boundary at $y^{*}=0$ and $-\infty<x^{*}<\infty$ (hereafter, asterisks denote dimensional quantities). The boundary is actuated with small-amplitude time-harmonic oscillations, prescribed by the time $t^{*}$ and position $x^{*}$ distribution of its normal velocity component,

$$
V_{w}^{*}\left(t^{*}, x^{*}\right)=\varepsilon U_{\mathrm{th}}^{*} v_{w}\left(x^{*}\right) \cos \left(\omega^{*} t^{*}\right) .
$$

In Eq. (1), $U_{\mathrm{th}}^{*}=\sqrt{2 \mathcal{R}^{*} T_{0}^{*}}$ denotes the mean thermal speed of a gas molecule (where $\mathcal{R}^{*}$ is the specific gas constant and $T_{0}^{*}$ is the reference gas temperature), $v_{w}\left(x^{*}\right)$ marks the nondimensional $\left(U_{\mathrm{th}}^{*}\right.$-scaled) $x^{*}$ variation of the wall normal velocity amplitude, and $\omega^{*}$ is the time frequency of imposed oscillations. It is assumed that $\varepsilon \ll 1$, so that the gas response may be linearized about its 


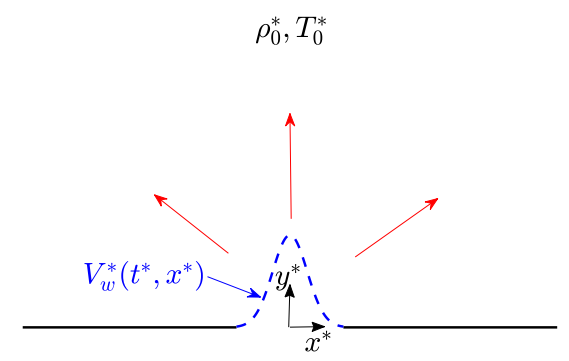

FIG. 1. Schematic of the problem: A semi-infinite gas layer, nominally set at thermodynamic equilibrium, is confined by an infinite fully diffuse planar wall at $x^{*}=0$. The wall is actuated with prescribed smallamplitude time- and $x^{*}$-dependent normal velocity profile, $V_{w}^{*}=V_{w}^{*}\left(t^{*}, x^{*}\right)$.

stationary equilibrium state of uniform density $\rho_{0}^{*}$ and temperature $T_{0}^{*}$. The wall is modeled as fully diffuse with a fixed temperature $T_{0}^{*}$.

The paper analyzes the effect of gas rarefaction on the two-dimensional propagation of acoustic waves generated by the wall excitation specified in Eq. (1). Focusing on localized wall displacements confined to the vicinity of the origin $x^{*}=0$, the level of gas rarefaction is governed by the ratio between the time frequency of imposed oscillations $\omega^{*}$ and the mean collision frequency of a gas molecule, $v_{0}^{*} \approx U_{\mathrm{th}}^{*} / l^{*}$, where $l^{*}$ denotes the gas molecular mean free path. The limit-case regimes of highly rarefied (collisionless) and continuum flows are expected to prevail at large and low values of $\omega^{*} l^{*} / U_{\mathrm{th}}^{*}$, respectively. Setting $\omega^{*-1}$ and $U_{\mathrm{th}}^{*}$ as the problem characteristic time and velocity scales, respectively, the consequent lengthscale is of the order of the acoustic-signal wavelength, $U_{\mathrm{th}}^{*} / \omega^{*}$. The problem is then governed by

$$
\mathrm{Kn}=\omega^{*} l^{*} / U_{\mathrm{th}}^{*} \text { and } v_{w}(x),
$$

denoting the system mean Knudsen number and prescribed wall vibrations amplitude, respectively. The nondimensional description is completed by taking $\rho_{0}^{*}$ and $T_{0}^{*}$ as the reference gas density and temperature, respectively.

In what follows, Secs. III and IV analyze the specific limits of highly rarefied and continuum limit conditions, expected at $\mathrm{Kn} \gg 1$ and $\mathrm{Kn} \ll 1$, respectively. The DSMC scheme, applied for counterpart numerical analysis of the problem, is discussed in Sec. V.

\section{FREE-MOLECULAR LIMIT}

Within the framework of gas kinetic theory and the present two-dimensional setup, the gas state is governed by the probability density function $f=f(t, x, y, \xi)$ of finding a gas molecule with velocity about $\xi=\left(\xi_{x}, \xi_{y}, \xi_{z}\right)$ at a position near $(x, y)$ at time $t$. At the linearized conditions assumed we write

$$
f(t, x, y, \xi)=F[1+\varepsilon \phi(t, x, y, \xi)],
$$

where $F=\pi^{-3 / 2} \exp \left[-\xi^{2}\right]$ denotes the nondimensional Maxwellian equilibrium distribution, and $\phi(t, x, y, \xi)$ marks the probability perturbation function [28]. Assuming the Knudsen number to be large, we consider the collisionless two-dimensional unsteady Boltzmann equation for $\phi(t, x, y, \xi)$ :

$$
\frac{\partial \phi}{\partial t}+\xi_{x} \frac{\partial \phi}{\partial x}+\xi_{y} \frac{\partial \phi}{\partial y}=0 .
$$

The free-molecular problem is amenable to a closed-form solution for an arbitrary (small-amplitude) source time dependence, $V_{w}=V_{w}(t, x)$. We therefore consider here the more general problem that does not a priori assume time-harmonic wall oscillations. Equation (4) is subject to a far-field decay condition together with a half-range fully diffuse boundary condition at the wall. After linearization, 
the latter takes the form

$$
\phi(t, x, 0, \boldsymbol{\xi} \cdot \hat{\mathbf{y}}>0)=\rho_{w}(t, x)+2 \xi_{y} V_{w}(t, x),
$$

where $\hat{\mathbf{y}}$ is a unit vector directed in the positive $y$ direction (normal to the boundary) and $\rho_{w}(t, x)$ is yet unknown. The solution for Eq. (4) subject to Eq. (5) is

$$
\phi(t, x, y, \boldsymbol{\xi})= \begin{cases}0, & \xi_{y} \leqslant 0 \\ \rho_{w}\left(t-y / \xi_{y}, x-y \xi_{x} / \xi_{y}\right)+2 \xi_{y} V_{w}\left(t-y / \xi_{y}, x-y \xi_{x} / \xi_{y}\right), & \xi_{y}>0 .\end{cases}
$$

To determine the wall function $\rho_{w}(t, x)$ appearing in Eq. (6), we make use of Eq. (3) and impose the linearized form of the impermeability condition on the normal velocity component $v(t, x, y)$,

$$
v(t, x, 0)=\frac{1}{\pi^{3 / 2}} \int_{-\infty}^{\infty} \xi_{y} \phi(t, x, 0, \xi) \exp \left[-\xi^{2}\right] d \xi=V_{w}(t, x)
$$

Substituting Eq. (6) into Eq. (7), we find

$$
\rho_{w}(t, x)=\sqrt{\pi} V_{w}(t, x),
$$

indicating the local dependence of the gas mass flux at the wall on its instantaneous velocity. Having solved for $\phi(t, x, y, \xi)$, appropriate quadratures of Eq. (3) over the velocity space yield expressions for the $\varepsilon$-scaled density $\rho(t, x, y)$; tangential and normal velocity components $u(t, x, y)$ and $v(t, x, y)$, respectively; and acoustic pressure $p(t, x, y)$ perturbations. Specifically, denoting

$$
I_{\xi_{j}}^{(n)}(t, x, y)=\frac{1}{\pi} \int_{-\infty}^{\infty} \int_{0}^{\infty} \xi_{j}^{n}\left(\sqrt{\pi}+2 \xi_{y}\right) V_{w}\left(t-\frac{y}{\xi_{y}}, x-\frac{\xi_{x}}{\xi_{y}} y\right) \exp \left[-\xi_{x}^{2}-\xi_{y}^{2}\right] d \xi_{y} d \xi_{x},
$$

the perturbation fields are expressed as

$$
\rho(t, x, y)=I_{\xi_{x}}^{(0)}, \quad u(t, x, y)=I_{\xi_{x}}^{(1)}, \quad v(t, x, y)=I_{\xi_{y}}^{(1)} \quad \text { and } \quad p(t, x, y)=\frac{1}{3}\left(2 I_{\xi_{x}}^{(2)}+2 I_{\xi_{y}}^{(2)}+I_{\xi_{x}}^{(0)}\right) .
$$

The temperature perturbation may be obtained using the linearized form of the equation of state,

$$
T(t, x, y)=p(t, x, y)-\rho(t, x, y) .
$$

Explicit expressions for the perturbation fields at the wall $(y=0)$ may be consequently obtained for an arbitrary choice of $V_{w}(t, x)$. For the density, $x$ velocity, and pressure, we obtain

$$
\rho(t, x, 0)=\frac{\pi+2}{2 \sqrt{\pi}} V_{w}(t, x), \quad u(t, x, 0)=0, \quad \text { and } p(t, x, 0)=\frac{3 \pi+8}{6 \sqrt{\pi}} V_{w}(t, x),
$$

respectively. Notably, the tangential velocity component vanishes at the wall. This is a consequence of averaging over the molecular velocities of the particles right before and right after colliding with the boundary, both maintaining Maxwellian distributions with a vanishing $x$-velocity component. As discussed later on, this contributes to the non-mono-polar radiation pattern obtained at freemolecular conditions, where no sound is propagating along the wall surface (see Sec. VI). Specific expressions for the free-molecular space and time distributions of the acoustic fields are discussed in Sec. VI for particular choices of $V_{w}(t, x)$.

\section{CONTINUUM LIMIT}

The problem at small Knudsen numbers is next analyzed based on a first-order "slip-flow" continuum-limit model consisting of the continuum Navier-Stokes-Fourier (NSF) balance equations and respective wall and far-field conditions. The model is first presented, after which its compressible ideal-flow $(\mathrm{Kn} \rightarrow 0$ ) limit solution is derived (Sec. IV A). The problem analysis at small yet finite $\mathrm{Kn} \ll 1$ is considered in Sec. IV B. 
Adopting the scaling introduced in Sec. II, the linearized two-dimensional NSF equations consist of the balances of mass,

$$
\frac{\partial \rho}{\partial t}+\frac{\partial u}{\partial x}+\frac{\partial v}{\partial y}=0
$$

$x$ momentum,

$$
\frac{\partial u}{\partial t}=-\frac{1}{2}\left(\frac{\partial \rho}{\partial x}+\frac{\partial T}{\partial x}\right)+\widetilde{K n}\left(\frac{4}{3} \frac{\partial^{2} u}{\partial x^{2}}+\frac{\partial^{2} u}{\partial y^{2}}+\frac{1}{3} \frac{\partial^{2} v}{\partial x \partial y}\right),
$$

$y$ momentum,

$$
\frac{\partial v}{\partial t}=-\frac{1}{2}\left(\frac{\partial \rho}{\partial y}+\frac{\partial T}{\partial y}\right)+\widetilde{K n}\left(\frac{\partial^{2} v}{\partial x^{2}}+\frac{4}{3} \frac{\partial^{2} v}{\partial y^{2}}+\frac{1}{3} \frac{\partial^{2} u}{\partial x \partial y}\right),
$$

and energy,

$$
\frac{\partial T}{\partial t}=-\frac{\gamma \widetilde{\mathrm{Kn}}}{\operatorname{Pr}}\left(\frac{\partial^{2} T}{\partial x^{2}}+\frac{\partial^{2} T}{\partial y^{2}}\right)-(\gamma-1)\left(\frac{\partial u}{\partial x}+\frac{\partial v}{\partial y}\right),
$$

where the linearized form of the equation of state for an ideal gas, $p=\rho+T$, has been applied. In Eqs. (14) and (15), the viscosity-based Knudsen number,

$$
\widetilde{\mathrm{Kn}}=\frac{\omega_{0}^{*} \mu_{0}^{*}}{\rho_{0}^{*} U_{\mathrm{th}}^{* 2}},
$$

is introduced, where $\mu_{0}^{*}$ denotes the gas dynamic viscosity at the reference temperature $T_{0}^{*}$. Considering a hard-sphere gas kinetic model, we fix $\mu_{0}^{*}=(5 \sqrt{\pi} / 16) \rho_{0}^{*} U_{\text {th }} l^{*}$ [29], yielding $\widetilde{\mathrm{Kn}}=$ $(5 \sqrt{\pi} / 16) \mathrm{Kn}$ [cf. Eq. (2)]. Also appearing in Eq. (15) are the gas Prandtl number, Pr, and ratio of specific heats, $\gamma$, which equal $2 / 3$ and $5 / 3$, respectively, in an ideal monatomic gas. Equations (12)-(15) are supplemented by the wall impermeability condition,

$$
v(t, x, 0)=V_{w}(t, x)
$$

together with first-order velocity slip,

$$
u(t, x, 0)=c_{u}\left(\frac{\partial u}{\partial y}+\frac{\partial v}{\partial x}\right)_{(t, x, 0)}+\left.c_{T}^{(1)} \frac{\partial T}{\partial x}\right|_{(t, x, 0)},
$$

and temperature jump,

$$
T(t, x, 0)=\left.c_{T}^{(2)} \frac{\partial T}{\partial y}\right|_{(t, x, 0)},
$$

conditions, as formulated by Aoki et al. [30]. A temperature jump component contributed by the normal stress at the wall $(\propto \partial v / \partial y)$ is not included in Eq. (19), as it is of higher order in the present linearized setup [see Eq. (118) in Ref. [30], et seq.]. Assuming a hard-sphere gas model,

$$
c_{u} \approx 1.111 \mathrm{Kn}, \quad c_{T}^{(1)} \approx 0.573 \mathrm{Kn}, \text { and } c_{T}^{(2)} \approx 2.127 \mathrm{Kn} .
$$

Far-field $(x, y \rightarrow \infty)$ decay conditions are additionally imposed on all perturbations.

In line with the harmonic time dependence of the wall forcing signal [see Eq. (1)], we assume sinusoidal time variation of all fields,

$$
F(t, x, y)=\operatorname{Re}\{\tilde{F}(x, y) \exp [i t]\} .
$$

Further, since the flow domain is infinite in the $x$ direction, it is useful to apply the $x$-Fourier transform,

$$
\bar{F}(k, y)=\int_{-\infty}^{\infty} \tilde{F}(x, y) \exp [-i k x] d x
$$


to the above problem. The system of Eqs. (12)-(15) is then transformed into

$$
\begin{aligned}
& i \bar{\rho}+i k \bar{u}+\bar{v}^{\prime}=0, \quad i \bar{u}=-\frac{i k}{2}(\bar{\rho}+\bar{T})+\frac{5 \mathrm{Kn} \sqrt{\pi}}{16}\left(\bar{u}^{\prime \prime}-\frac{4 k^{2}}{3} \bar{u}+\frac{i k}{3} \bar{v}^{\prime}\right), \\
& i \bar{v}=-\frac{1}{2}\left(\bar{\rho}^{\prime}+\bar{T}^{\prime}\right)+\frac{5 \mathrm{Kn} \sqrt{\pi}}{16}\left(\frac{i k}{3} \bar{u}^{\prime}+\frac{4}{3} \bar{v}^{\prime \prime}-k^{2} \bar{v}\right), \\
& i \bar{T}=-\frac{25 \mathrm{Kn} \sqrt{\pi}}{32}\left(\bar{T}^{\prime \prime}-k^{2} \bar{T}\right)-\frac{2}{3}\left(i k \bar{u}+\bar{v}^{\prime}\right),
\end{aligned}
$$

where primes denote derivatives in the $y$ direction. These are supplemented by the transformed form of the boundary conditions in Eqs. (17)-(20),

$$
\bar{v}(k, 0)=\bar{V}_{w}(k), \quad \bar{u}(k, 0)=\left[c_{u}\left(\bar{u}^{\prime}+i k \bar{v}\right)+i k c_{T}^{(1)} \bar{T}\right]_{(k, 0)} \quad \text { and } \quad \bar{T}(k, 0)=c_{T}^{(2)} \bar{T}^{\prime}(k, 0) .
$$

The analysis of the $k$-plane problem is described in Sec. IV B. Once completed, the solution in the physical $(t, x, y)$ domain is obtained by applying the inverse transformation,

$$
F(t, x, y)=\frac{1}{2 \pi} \operatorname{Re}\left\{\exp [i t] \int_{-\infty}^{\infty} \bar{F}(k, y) \exp [i k x] d k\right\},
$$

to each of the hydrodynamic perturbations. Before considering the general slip-flow solution, we discuss the ideal-flow $(\mathrm{Kn} \rightarrow 0)$ problem in Sec. IV A.

\section{A. Ideal-flow limit}

Assuming $\mathrm{Kn} \rightarrow 0$ in Eq. (23), the problem degenerates into its ideal compressible (Euler) flow limit, where viscous and heat-conduction effects are neglected. Here, the dynamic and thermodynamic flow descriptions are decoupled, and the wall slip and thermal boundary conditions cannot be imposed. The hydrodynamic equations may be cast into a single equation for the flow velocity potential, forming the Helmholtz $k$-plane equivalent of the linear wave equation. The transformed normal velocity accordingly satisfies

$$
\bar{v}^{\prime \prime}+\lambda^{2} \bar{v}=0
$$

where

$$
\lambda(k)=\sqrt{\frac{6-5 k^{2}}{5}} .
$$

Imposing a nondiverging condition at $y \rightarrow \infty$ and the impermeability condition in Eq. (24), we obtain the particular solution

$$
\bar{v}(k, y)=\bar{v}_{w}(k) \exp [i \lambda y],
$$

and consequent expressions for all other transformed perturbations:

$$
\begin{aligned}
& \bar{u}(k, y)=\frac{k}{\lambda} \bar{v}_{w}(k) \exp [i \lambda y], \quad \bar{\rho}(k, y)=-\frac{6}{5 \lambda} \bar{v}_{w}(k) \exp [i \lambda y], \\
& \bar{T}(k, y)=\frac{2}{3} \bar{\rho}(k, y) \text { and } \bar{p}(k, y)=\frac{5}{3} \bar{\rho}(k, y) .
\end{aligned}
$$

The multiplying-factor dependence between the density and temperature perturbations reflects the isentropic relation, $T=(\gamma-1) \rho=2 \rho / 3$, effective at ideal-flow conditions. The hydrodynamic fields in the $(t, x, y)$ plane are calculated using Eq. (25). The solution obtained thereby will be applied in Sec. VI to illustrate the contrast between the system behavior in the continuum and free-molecular limits. 


\section{B. Slip-flow limit}

Assuming nonzero $\mathrm{Kn} \ll 1$ values, the problem in Eqs. (23) and (24) is considered. To this end, Eq. (23) is cast as a system of coupled first-order equations,

$$
\mathbf{g}^{\prime}=\mathrm{Ag},
$$

where $\mathbf{g}=\left[\bar{u}, \bar{u}^{\prime}, \bar{v}, \bar{v}^{\prime}, \bar{T}, \bar{T}^{\prime}\right]^{\mathrm{T}}$ is the vector of unknown functions and $\mathrm{A}=\mathrm{A}(k, \mathrm{Kn})$ is the matrix of coefficients. The general solution for Eq. (30) is

$$
\mathbf{g}=\Sigma_{n=1}^{6} D_{n} \mathbf{v}_{\mathbf{n}} \exp \left[\lambda_{n} y\right]
$$

where $\mathbf{v}_{\mathbf{n}}(k, \mathrm{Kn})$ and $\lambda_{n}(k, \mathrm{Kn})$ mark the $n=1, \ldots, 6$ eigenvectors and eigenvalues of the matrix A, respectively, and $D_{n}$ are the unknown scalar coefficients multiplying the respective $\mathbf{v}_{\mathbf{n}} \exp \left[\lambda_{n} y\right]$ components. Analysis of the characteristic sixth-order polynomial of the matrix $A$ at $\mathrm{Kn} \ll 1$ indicates that its roots $\lambda_{n}$ consist of three complex pairs of alternating signs. Omitting the three eigenvalues with $\operatorname{Re}\left\{\lambda_{n}\right\}>0$ to satisfy the far-field decay condition, the $\mathrm{Kn} \ll 1$ approximations for the remaining $\lambda_{1,2,3}$ are

$$
\begin{aligned}
& \lambda_{1}=i \sqrt{\frac{6-5 k^{2}}{5}}\left[1-\frac{21 i \sqrt{\pi}}{8\left(6-5 k^{2}\right)} \mathrm{Kn}+O\left(\mathrm{Kn}^{2}\right)\right], \\
& \lambda_{2}=-\frac{4 \sqrt{i}}{\sqrt{5 \pi^{1 / 2} \mathrm{Kn}}}\left[1-\frac{5 \sqrt{\pi}}{32} k^{2} \mathrm{Kn}+O\left(\mathrm{Kn}^{2}\right)\right] \\
& \text { and } \lambda_{3}=-\frac{4 \sqrt{2 i}}{\sqrt{15 \pi^{1 / 2} \mathrm{Kn}}}\left[1-\frac{5 i \sqrt{\pi}}{16}\left(\frac{3 k^{2}}{4}-\frac{1}{15}\right) \mathrm{Kn}+O\left(\mathrm{Kn}^{2}\right)\right] .
\end{aligned}
$$

As $\mathrm{Kn} \rightarrow 0, \lambda_{1}$ recovers its ideal-flow limit, $i \lambda=i \sqrt{\left(6-5 k^{2}\right) / 5}$, obtained in Eq. (28). The $\lambda_{2}$ and $\lambda_{3}$ eigenvalues, as well as the $O(\mathrm{Kn})$ correction to $\lambda_{1}$, reflect viscous and heat conduction effects, inevitably missing at ideal-flow conditions. With decreasing $\mathrm{Kn} \rightarrow 0$, the contributions of the $\lambda_{2,3}$ modes become confined to the immediate vicinity of the solid wall, as $\operatorname{Re}\left\{\lambda_{2,3}\right\} \propto-\mathrm{Kn}^{-1 / 2}$. With increasing $\mathrm{Kn}$, these eigenmodes affect a wider gas layer, resulting in deviations between the Euler and NSF flow descriptions.

To specify the particular solution in Eq. (31), the coefficients $D_{n}$ multiplying $\mathbf{v}_{\mathbf{n}} \exp \left[\lambda_{n} y\right]$ in Eq. (31) $(n=1,2,3)$ are calculated through the imposition of the impermeability, slip, and jump wall conditions in Eq. (24). The result in the $(t, x, y)$ physical domain is then obtained using Eq. (25).

\section{NUMERICAL SCHEME: DSMC METHOD}

The DSMC method, initially proposed by Bird [31], is a stochastic particle method commonly applied for the analysis of rarefied gas flows. In the present paper, we make use of the DSMC scheme to examine the free-molecular and continuum limit solutions obtained in Secs. III and IV, respectively, and to capture the system behavior at intermediate Knudsen numbers. We accordingly adopt Bird's algorithm, together with the hard-sphere model of molecular interaction, to simulate the gas state. In line with the problem formulation, the wall surface is assumed fully diffuse, with a fixed temperature and a prescribed $x^{*}$-dependent time-harmonic normal velocity profile. To simulate the boundary condition yet maintain the surface fixed (which considerably simplifies the calculation), an algorithm for adding or subtracting particles has been applied, ensuring that the number flux of gas particles emitted at the wall agrees with the flux required to satisfy the impermeability condition. The algorithm is described below.

Considering the linearized problem, the difference between the reflected and incoming particles number flux at the wall is equal to $\rho_{0}^{*} v_{w}^{*}\left(x^{*}\right) / m^{*}$, where $m^{*}$ denotes the particle molecular mass. Assuming a simulated wall of length $L_{w}^{*}$ actuated at a uniform velocity with a maximal amplitude $v_{w}^{* \max }$ during a time step $\Delta t^{*}$, the difference between the total number of outgoing and incoming 
particles is

$$
N_{\text {diff }}=N_{\text {out }}-N_{\text {in }}=\frac{\rho_{0}^{*}}{m^{*}} v_{w}^{* \max } \triangle t^{*} L_{w}^{*} .
$$

To simulate the present two-dimensional $x^{*}$-dependent (nonuniform) distribution of $v_{w}^{*}\left(x^{*}\right)$, an acceptance-rejection scheme has been applied for adding or deleting particles. In a case where the difference in Eq. (33) is positive, $N_{\text {diff }}$ attempts were made to add particles to the simulation domain. In each attempt, the boundary velocity $v_{w}^{*}\left(x_{p}^{*}\right)$ at the particle impingement location $x_{p}^{*}$ was calculated and a particle was added if

$$
\frac{v_{w}^{*}\left(x_{p}^{*}\right)}{v_{w}^{* \max }}>R_{U},
$$

where $R_{U} \in[0,1]$ denotes a uniformly distributed random number. If added, the particle was given a velocity in accordance with the diffuse reflection distribution. In the opposite case where $N_{\text {diff }}<0$, particles that impinged the boundary were subject to a similar acceptance-rejection scheme for their removal. Here, the wall velocity at the particle's impingement location was calculated and the scheme in Eq. (34) was used to decide whether the particle should be omitted from the domain.

Each computation was started from an initial equilibrium state and followed in time until a final periodic state was reached, typically after two to three oscillation periods. To mimic the formulated half-space problem, a rectangular computational domain was set, with virtual "side" and "top" boundaries placed far enough to not affect the system response. In practice, placing these boundaries at $x_{\mathrm{sim}}^{*}, y *_{\mathrm{sim}} \approx 10-15 U_{\mathrm{th}}^{*} / \omega^{*}$ has proved sufficiently far to neglect their impact at $\mathrm{Kn} \gtrsim 0.1$. At lower Knudsen numbers, however, the signal decay rate was found too low to neglect the influence of sound reflection by the virtual boundaries (see Sec. VI). While this could be overcome through an increase in the computation domain size, it would have made the calculation at small Knudsen numbers prohibitively expensive. This limitation, hindering a quantitative comparison between the continuum-limit and DSMC results at Kn $\ll 1$, is illustrated in Sec. VI (see Figs. 6 and 7).

In a typical calculation, the domain was divided into $3-6 \times 10^{3}$ cells. An additional division of each cell into collisional subcells was carried out to comply with the mean-free-path limitations. The subcells size was set to $l^{*} / 4$ and the simulation time step was taken $1 / 4 \times l^{*} / U_{\mathrm{th}}^{*}$. A typical run consisted of $\approx 4 \times 10^{7}$ particles, with $\approx 200$ realizations carried to sufficiently reduce the numerical noise. Each simulation lasted from several hours in the free-molecular limit up to a week in the continuum limit using a ten-core Intel i7-6950 machine. In line with the linearized problem formulation, a value of $\varepsilon=0.05$ was taken, for which nonlinear effects were found negligible.

Having described the above DSMC algorithm, it is noted that previous studies have developed particular low-variance DSMC schemes that may be better suited for analyzing the present linearized [32] and time-periodic [33] setup. Yet, for the purpose of validating our analytical-limit results and illustrating the system behavior at intermediate-flow conditions, we find the current scheme satisfactory. Additionally, the present computation has the advantage of not assuming (and thus not being limited to) small signal amplitudes or time periodic behavior of the solution. In this sense, it provides a more general description that examines the validity of these assumptions, namely, the significance of system nonlinearity at a given signal amplitude, and the system convergence to harmonic solution.

\section{RESULTS}

Focusing on the time-harmonic wall oscillations as specified in Eq. (1), we present our results for specific choices of the $x$ distribution of the wall velocity amplitude,

$$
v_{w}^{(\delta)}(x)=\delta(x) \text { and } v_{w}^{(G)}(x)=\exp \left[-\alpha x^{2}\right],
$$

corresponding to dirac-delta and Gaussian excitations, respectively. While the dirac-delta response of the system is amenable to a closed-form solution in the free-molecular and continuum limits, its 
DSMC realization appears ambiguous. We therefore consider also the gas response to a Gaussian wall perturbation (where $\alpha$ is a fixed parameter), for the purpose of numerical validation and illustration of the system behavior at intermediate $\mathrm{Kn} \sim O(1)$ numbers. The delta-function and Gaussian excitation gas responses are discussed in Secs. VI A and VI B, respectively.

\section{A. Delta-function excitation}

Starting with the free-molecular system response, we substitute $v_{w}^{(\delta)}(x)=\delta(x)$ into the nondimensional $\varepsilon$-scaled counterpart of Eq. (1) and then into Eqs. (9) and (10). Carrying out the $\xi_{x}$ quadratures explicitly, we find

$$
\begin{aligned}
& \rho_{\mathrm{FM}}^{(\delta)}(t, x, y)=\frac{1}{\pi y} \int_{0}^{\infty} \xi_{y}\left(\sqrt{\pi}+2 \xi_{y}\right) \exp \left[i\left(t-\frac{y}{\xi_{y}}\right)-\left(1+\frac{x^{2}}{y^{2}}\right) \xi_{y}^{2}\right] d \xi_{y}, \\
& u_{\mathrm{FM}}^{(\delta)}(t, x, y)=\frac{x}{\pi y^{2}} \int_{0}^{\infty} \xi_{y}^{2}\left(\sqrt{\pi}+2 \xi_{y}\right) \exp \left[i\left(t-\frac{y}{\xi_{y}}\right)-\left(1+\frac{x^{2}}{y^{2}}\right) \xi_{y}^{2}\right] d \xi_{y}, \\
& v_{\mathrm{FM}}^{(\delta)}(t, x, y)=\frac{1}{\pi y} \int_{0}^{\infty} \xi_{y}^{2}\left(\sqrt{\pi}+2 \xi_{y}\right) \exp \left[i\left(t-\frac{y}{\xi_{y}}\right)-\left(1+\frac{x^{2}}{y^{2}}\right) \xi_{y}^{2}\right] d \xi_{y}, \\
& p_{\mathrm{FM}}^{(\delta)}(t, x, y)=\frac{2}{3 \pi y} \int_{0}^{\infty} \xi_{y}^{3}\left(\frac{x^{2}}{y^{2}}+1+\frac{1}{2 \xi_{y}^{2}}\right)\left(\sqrt{\pi}+2 \xi_{y}\right) \exp \left[i\left(t-\frac{y}{\xi_{y}}\right)-\left(1+\frac{x^{2}}{y^{2}}\right) \xi_{y}^{2}\right] d \xi_{y},
\end{aligned}
$$

where the real part should be taken to obtain the physical-space solution for the density, $x$-velocity, $y$-velocity, and pressure perturbation fields, respectively. The "FM" subscript denotes the freemolecular limit of the solution. Inspecting the above expressions, we find that the density, $y$ velocity, and acoustic pressure are even functions of $x$, while the $x$ velocity is odd, indicating that the disturbance propagates symmetrically about $x=0$. Additionally, all even perturbations obtain their maximum absolute value at $x=0$ (at given $t$ and $y$ ), as indicated by the exponential $x$ dependence of their respective integrands. Along the wall and excluding the source location (i.e., for $y=0$ and $x \neq 0$ ) all fields vanish due to their $\exp \left[-x^{2} / y^{2}\right]$ factor, in agreement with the expressions in Eq. (11) for $V_{w}(t, x)=\delta(x) \cos (t)$. Specifically, no pressure disturbance is radiated along the wall (perpendicular to the axis of source oscillations) in the free-molecular limit.

Traversing to the $\mathrm{Kn} \rightarrow 0$ ideal-flow limit, we substitute the Fourier-transformed $\bar{v}_{w}^{(\delta)}(k)=1$ into Eqs. (28) and (29) and then into Eq. (25), to obtain

$$
\begin{aligned}
& \rho_{\mathrm{Eu}}^{(\delta)}(t, x, y)=-\frac{3}{5 \pi} \int_{-\infty}^{\infty} \frac{1}{\lambda} \exp [i(t+k x+\lambda y)] d k, \\
& u_{\mathrm{Eu}}^{(\delta)}(t, x, y)=\frac{1}{2 \pi} \int_{-\infty}^{\infty} \frac{k}{\lambda} \exp [i(t+k x+\lambda y)] d k, \\
& v_{\mathrm{Eu}}^{(\delta)}(t, x, y)=\frac{1}{2 \pi} \int_{-\infty}^{\infty} \exp [i(t+k x+\lambda y)] d k, \\
& p_{\mathrm{Eu}}^{(\delta)}(t, x, y)=-\frac{1}{\pi} \int_{-\infty}^{\infty} \frac{1}{\lambda} \exp [i(t+k x+\lambda y)] d k .
\end{aligned}
$$

As in Eq. (36), the real part in each field should be taken. The "Eu" subscript denotes the Eulerflow limit of the solution. The following discussion on the differences between the free-molecular and continuum-limit results relies on numerical and asymptotic evaluations of the quadratures in Eqs. (36) and (37).

Figure 2 presents colormap snapshots at quarter period $(t=\pi / 2)$ of the $\varepsilon$-scaled acoustic pressure and $x$-velocity component in response to a time-harmonic delta-function excitation. The 

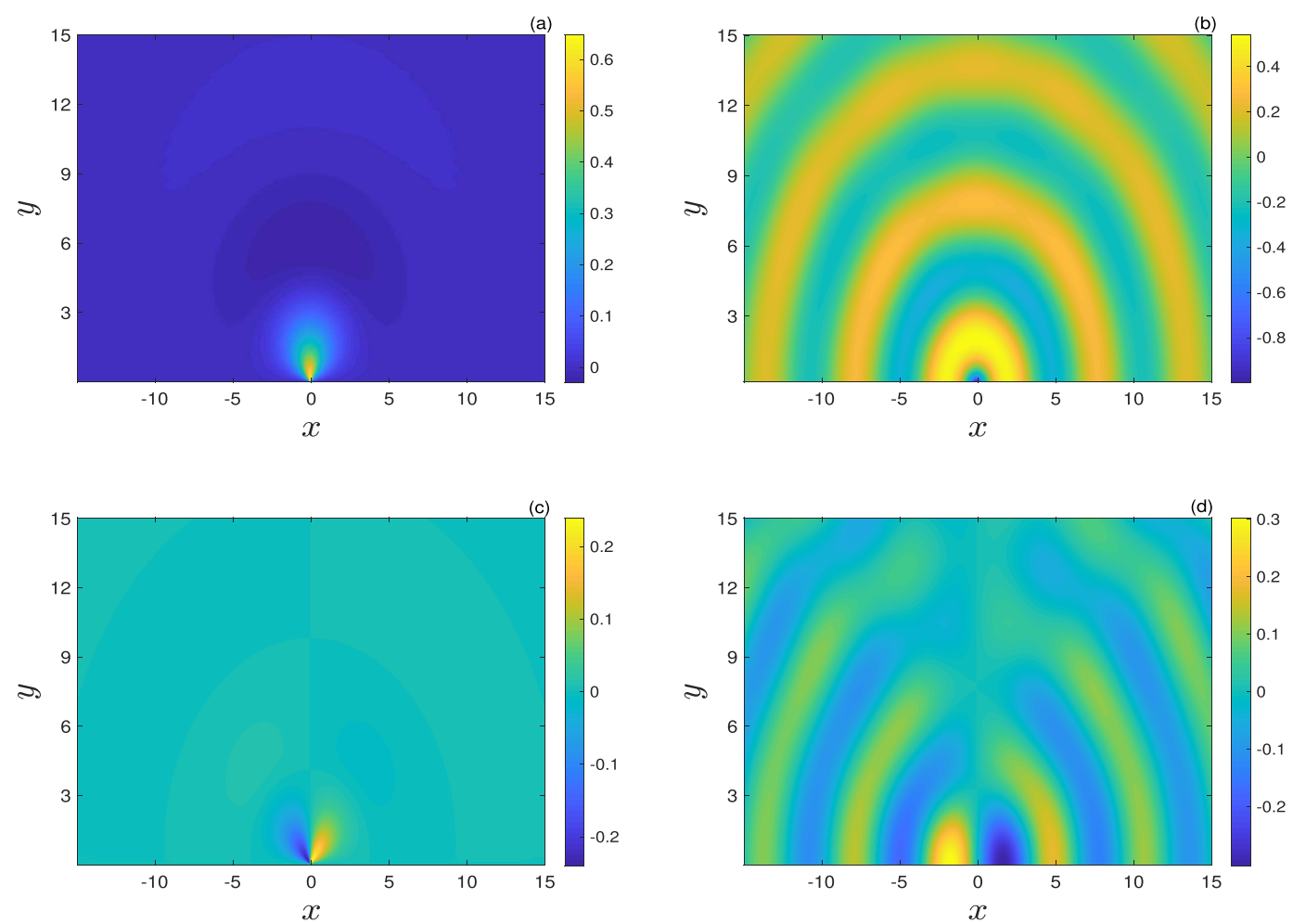

FIG. 2. Colormaps of the $\varepsilon$-scaled instantaneous (a), (b) acoustic pressure and (c), (d) $x$-velocity component at quarter period $(t=\pi / 2)$ in response to a delta function excitation in the (a), (c) free-molecular and (b), (d) ideal-flow limits.

figure compares between the free-molecular [Figs. 2(a) and 2(c)] and ideal-flow [Figs. 2(b) and 2(d)] system responses. Remarkably, distinct differences are observed between the two limits, in both directivity and decay rate of the acoustic signal. In the continuum limit, the signal propagates isotropically from the source in a monopole-type pattern with slowly decaying oscillations that remain significant within the presented $(-15 \leqslant x \leqslant 15,0 \leqslant y \leqslant 15)$ zone. At free-molecular conditions, a nonisotropic propagation pattern is viewed, achieving its maximum along the $y$ direction (the axis of wall oscillations) and vanishing in the $x$ direction along the wall. The free-molecular signal is rapidly diminishing, penetrating to only $y \lesssim 5$ acoustic wavelengths in the $y$ direction (and to smaller distances in other directions) into the gas. The even and odd characteristics of the pressure and $x$-velocity fields, respectively, expected from problem symmetry and the expressions in Eqs. (36) and (37), are observed.

To gain further insight into the above differences, Fig. 3 compares between the directivity fields of the acoustic signals in the collisionless and ideal-flow limits. Focusing on the far-field acoustic pressure, the figure shows the variation of the scaled pressure amplitude, $|p| /\left|p_{\max }\right|$, with the azimuthal direction $\theta$ at a fixed distance $r \gg 1$ from the origin. Here, $\left|p_{\max }\right|$ marks the maximum value of the pressure amplitude at the chosen $r$ distance, so that $|p| /\left|p_{\max }\right| \leqslant 1$. The direction $\theta=0$ coincides with the positive $y$ axis, whereas $\theta=\pi / 2$ and $-\pi / 2$ mark the positive and negative $x$ directions, respectively. Our calculations indicate that the results in Fig. 3 are independent of the specific choice of $r$ for $r \gtrsim 1$, and that $\left|p_{\max }\right|$ invariably occurs at $\theta=0$.

Inspecting the results in Fig. 3, the differences between the continuum and free-molecular limits are evident. Indeed, at $\mathrm{Kn} \rightarrow 0$ the signal is emitted isotropically in all directions, as seen by the semicircle form of the solid black line, satisfying $|p| /\left|p_{\max }\right|=1$ for all $-\pi / 2 \leqslant \theta \leqslant \pi / 2$. The 


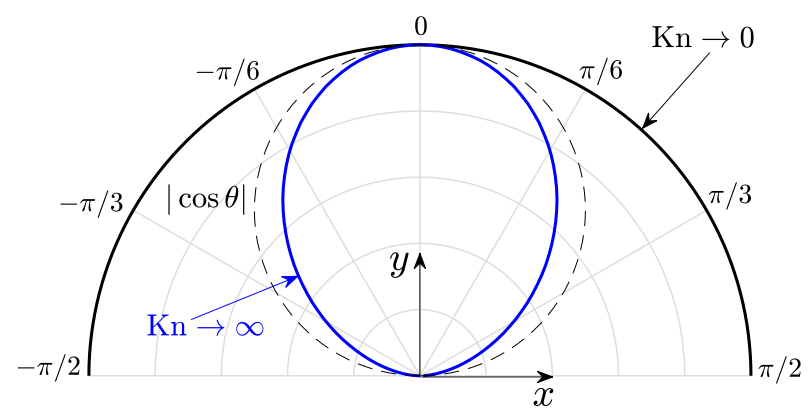

FIG. 3. Directivity map of the far-field scaled acoustic pressure amplitude, $|p| /\left|p_{\max }\right|$, in response to a delta function excitation at free-molecular (blue line) and ideal-flow (black curve) conditions. The thin dashed curve presents the directivity field of a dipole at continuum conditions, $|p| /\left|p_{\max }\right|=|\cos \theta|$. The half-circle black curve indicates monopolar radiation at $\mathrm{Kn} \rightarrow 0$, whereas the blue line depicts a "nearly-dipole" source pattern at $\mathrm{Kn} \rightarrow \infty$.

$\mathrm{Kn} \rightarrow \infty$ curve, however, shows a nonisotropic propagation pattern, with a maximum achieved along the $\theta=0$ axis, and a vanishing signal in the $\pm x$ directions. Equivalently supported by the expression for $p_{\mathrm{FM}}^{(\delta)}$ in Eq. (36), it is established that gas rarefaction qualitatively affects sound directivity in the current setup, turning it from a monopole-type to a "nearly dipole." Yet, an "exact" dipole pattern is not obtained here, as shown by comparison between the solid blue curve and the dashed black line in Fig. 3 (the latter marking the directivity $|p| /\left|p_{\max }\right|=|\cos \theta|$ of a dipole source at continuum conditions). Physically, such a pattern should not be anticipated here, and is added only for reference, as the model governing sound propagation at highly rarefied conditions is much different from the continuum limit. At the kinetic level, the tangential velocity in the free-molecular limit originates from the gradient in the molecular flux along the boundary, which is dominated by the imposed normal wall velocity. Specifically, as indicated by Eq. (11), the tangential gas velocity vanishes at the wall surface, contributing to the directivity pattern obtained. In the continuum limit, in contrast, normal momentum is inevitably transformed into tangential momentum through the mechanism of molecular collisions at all distances from the surface, leading to the calculated monopolar signal field.

Having discussed the acoustic field directivity, it is of additional interest to evaluate the acoustic decay rate at large distances from the source and rationalize the differences between the large- and small-Kn limits. Kinetically, in the free-molecular regime, the wall-reflected molecules are carrying information from the boundary with no intermolecular collisions taking place. Hence, molecules at a given location and time superpose the diverse wall states along a period at different retarded times. The disparity in these states is the cause for the strong signal decay observed in the $\mathrm{Kn} \rightarrow \infty$ limit. In the continuum limit, however, the molecules are traveling "ballistically" an average distance of one mean free path, which is much shorter than the acoustic wavelength. Their velocities are then much more "in-phase," leading to a lower decay rate of the signal.

To obtain a quantitative estimate of the signal decay rate, we consider the $y \gg 1$ limit of the free-molecular and ideal-flow acoustic fields specified in Eqs. (36) and (37), respectively. Starting with the far-field free-molecular response, we examine integrals of the form

$$
I_{n}(y)=\int_{0}^{\infty}\left(\sqrt{\pi}+2 \xi_{y}\right) \xi_{y}^{n} \exp \left[-i \frac{y}{\xi_{y}}-\xi_{y}^{2}\right] d \xi_{y} \quad \text { with } n=1,2, \ldots,
$$

where the $\exp \left[-x^{2} / y^{2}\right]$ Gaussian $x$ dependence appearing in Eq. (36) has been omitted for $x \sim O(1)$. This approximation holds for $x=0$, where all even fields attain their aforementioned maximum value, and should also be valid for $x \lesssim O(1) \ll y$. Following Abramowitz [34], the far-field leadingorder expressions for the hydrodynamic perturbations are obtained in Appendix A 1. Specifically, 

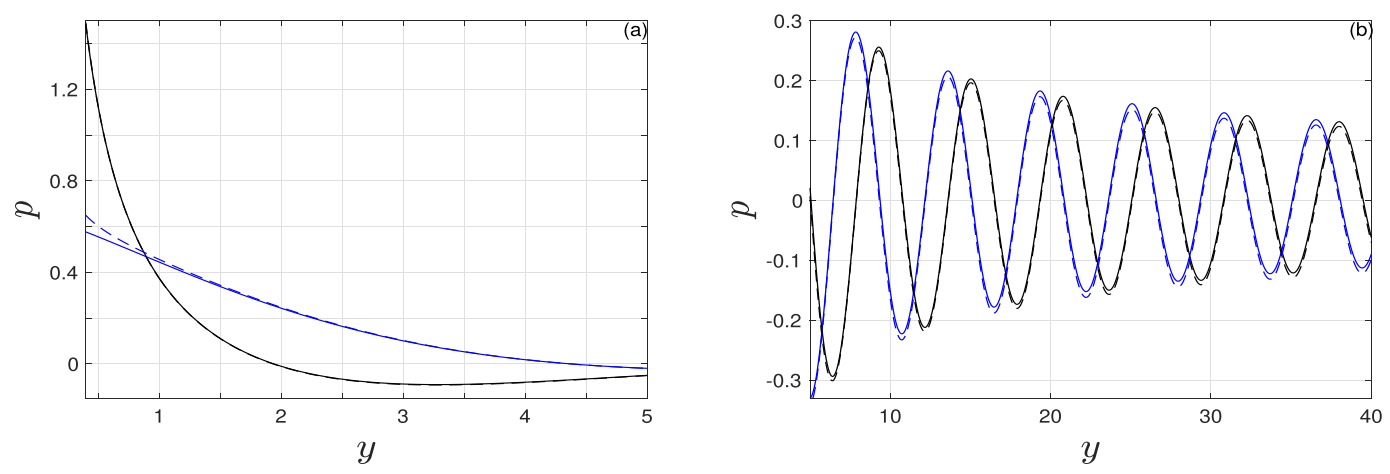

FIG. 4. Comparison between the $y$ variations of the integral-based (solid lines) and asymptotic (dashed curves) evaluations of the acoustic far field at $x=0$ in the (a) free-molecular and (b) ideal-flow limits in response to time-harmonic delta-function excitation. The black and blue curves depict the solutions at period and quarter period times, respectively.

the leading-order far-field acoustic pressure is estimated as

$$
p_{\mathrm{FM}}^{(\delta)}(t, x \sim O(1), y \gg 1) \approx \frac{-1+i \sqrt{3}}{2^{1 / 3} \sqrt{27 \pi}} y^{1 / 3} \exp \left[i t-\frac{3(1+i \sqrt{3})}{2^{5 / 3}} y^{2 / 3}\right],
$$

indicating that the far free-molecular pressure decay rate is $\sim O\left(y^{1 / 3} \exp \left[-3 y^{2 / 3} / 2^{5 / 3}\right]\right)$. In line with the analysis in Sec. III, it is noted that this estimate is limited to distances $y$ far from the source that are yet small compared with the gas kinetic mean free path $(y \ll \mathrm{Kn}$ in the present nondimensional formulation).

Turning to continuum-limit conditions, we examine Eq. (37) and seek for a far-field approximation of

$$
I(x, y)=\int_{-\infty}^{\infty} F(k) \exp [i(k x+\lambda y)] d k,
$$

where the harmonic $\exp [i t]$ time dependence has been omitted for convenience. Substituting $\lambda(k)=$ $\sqrt{\left(6-5 k^{2}\right) / 5}$ into Eq. (40) [see Eq. (27)] and making a change of variables $s=k \sqrt{5 / 6}$, the integral is recast in the form

$$
I(x, y)=\int_{-\infty}^{\infty} F(s) \exp \left[\sqrt{\frac{6}{5}} i\left(x s+y \sqrt{1-s^{2}}\right)\right] d s .
$$

Applying the method of stationary phase, the $y \gg 1$ limit of $I(x, y)$ at $x \sim O(1)$ is evaluated in Appendix A 2. Specifically, the far acoustic pressure is given by

$$
p_{\mathrm{Eu}}^{(\delta)}(t, x \sim O(1), y \gg 1) \approx-\sqrt{\frac{2 \sqrt{5}}{\pi y \sqrt{6}}} \exp \left[i \left(t+\sqrt{\left.\left.\frac{6}{5} y-\frac{\pi}{4}\right)\right],}\right.\right.
$$

indicating that it is oscillatory decaying at a rate $\sim O\left(y^{-1 / 2}\right)$ and a scaled wave number of $\sqrt{6 / 5}$. The latter originates from the ratio between the mean thermal speed (presently used as the problem velocity scale, $\left.U_{\mathrm{th}}^{*}=\sqrt{2 \mathcal{R}^{*} T_{0}^{*}}\right)$ and the mean speed of sound at continuum conditions $\left(=\sqrt{\gamma \mathcal{R}^{*} T_{0}^{*}}\right)$.

Comparing between Eq. (42) and the free-molecular decay rate in Eq. (39), the vanishing of the latter at significantly smaller distances from the source, viewed in Fig. 2, is quantitated. The validity of the far-field approximations for the free-molecular and continuum limit pressures is illustrated in Fig. 4, comparing between the $y \gg 1$ estimates and their integral-form counterparts in Eqs. (36) and (37). The stronger decay rate of the free-molecular pressure perturbation is noted again, vanishing 

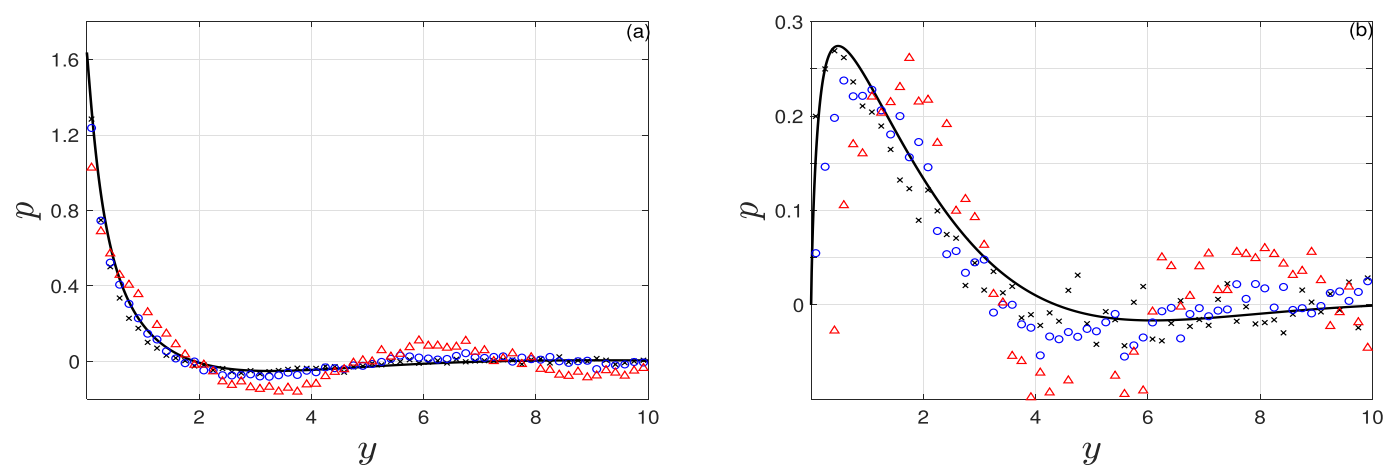

FIG. 5. Comparison between the collisionless-flow description (solid lines) and DSMC results at $\mathrm{Kn}=$ 5 (crosses), $\mathrm{Kn}=0.5$ (blue circles), and $\mathrm{Kn}=0.1$ (red triangles) for the acoustic pressure in response to Gaussian excitation with $\alpha=10$ along $x=0$ at (a) period $(t=2 \pi)$ and (b) quarter period $(t=\pi / 2)$ times.

at only a few acoustic wavelengths from the source. In Fig. 4(a), the asymptotic result for the freemolecular field contains the leading-order and first two correction terms (see Appendix A 1), and is in an excellent agreement with the integral-based result for $y \gtrsim 1$. In Fig. 4(b), slight deviations are observed between the solid and dashed curves, caused by higher-order corrections in the asymptotic calculation that were not evaluated here.

Considering the fundamentally disparate mathematical models governing the free-molecular (collisionless Boltzmann equation) and continuum (linear wave equation) limits, the qualitatively different results observed in Figs. 2 and 3 may have been anticipated. Yet, these differences could not be revealed in previous one-dimensional investigations of the problem, where unidirectional acoustic transmission was imposed. In particular, the two-dimensional study allows for the different characteristics of sound directivity to become effective, as demonstrated by Fig. 3 and its interpretation.

\section{B. Gaussian excitation}

As the gas response to a delta-function excitation was not modeled using DSMC calculations, the system response to Gaussian-distributed wall actuation is next considered. This is used to examine our limit-case solutions and illustrate the gas behavior at intermediate Knudsen numbers. We assume the wall velocity profile $v_{w}^{(G)}(x)=\exp \left[-\alpha x^{2}\right]$ specified in Eq. (35), and take $\alpha=10$ to focus on a source concentrated at $|x| \ll 1$. To calculate the free-molecular gas response, $v_{w}^{(G)}(x)$ is substituted into the nondimensional $\varepsilon$-scaled counterpart of Eq. (1) and then into Eqs. (9) and (10). Carrying out the $\xi_{x}$ quadratures, we obtain the integral expressions specified in Appendix B for the acoustic perturbations. In the continuum limit, we substitute the Fourier-transformed $\bar{v}_{w}^{(G)}(k)=$ $\sqrt{\pi / \alpha} \exp \left[-k^{2} /(4 \alpha)\right]$ into Eqs. (28) and (29) and then into Eq. (25) to formulate the integral expressions for the acoustic perturbations at ideal-flow conditions. The results in the slip-flow (NSF) regime follow the procedure outlined in Sec. IV B with the same form for $\bar{v}_{w}^{(G)}(k)$. The integrals, in both free-molecular and continuum limits, are evaluated numerically.

To examine the validity of the free-molecular solution, Fig. 5 compares between the acoustic pressure field at collisionless flow conditions, $p_{\mathrm{FM}}^{(G)}(t, x, y)$ [see Eq. (B1)], and DSMC results at different Knudsen numbers. The comparison is made at both period [Fig. 5(a)] and quarter period [Fig. 5(b)] times, where the pressure $y$ variation is presented at $x=0$. The agreement between the $\mathrm{Kn} \rightarrow \infty$ (solid lines) and $\mathrm{Kn}=5$ (crosses) results is good, in support of the validity of the former description at large enough Knudsen numbers. Yet, the DSMC signal is found noisier at quarter period time, where the wall velocity instantaneously vanishes and the pressure amplitude is relatively small. At $\mathrm{Kn}=0.5$, DSMC predictions (denoted by blue circles) show slight systematic 

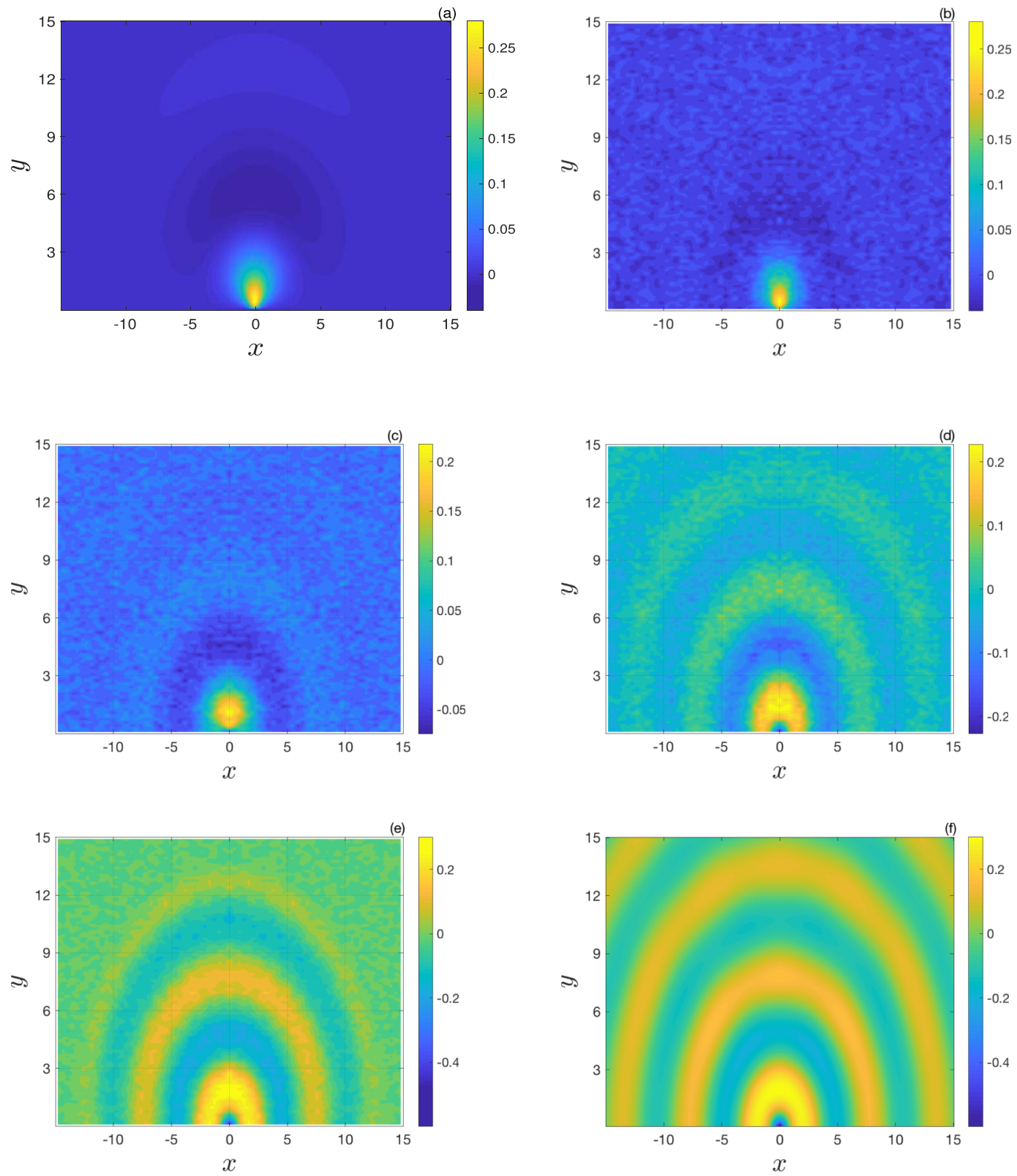

FIG. 6. Effect of the Knudsen number on the acoustic pressure in response to Gaussian excitation with $\alpha=10$ : time snapshots at quarter period $(t=\pi / 2$ ) with (a) $\mathrm{Kn} \rightarrow \infty$, (b) $\mathrm{Kn}=5$, (c) $\mathrm{Kn}=0.5$, (d) $\mathrm{Kn}=0.1$, (e) $\mathrm{Kn}=0.01$, and (f) $\mathrm{Kn} \rightarrow 0$. The results in Figs. 6(a) and 6(f) describe the analytical free-molecular and ideal-flow solutions, respectively, while Figs. 6(b)-6(e) present numerical DSMC computations.

deviations from the collisionless result. These deviations become more significant at $\mathrm{Kn}=0.1$, as depicted by the red triangles data. Indeed, the free-molecular description is expected to break down at $\mathrm{Kn} \lesssim 1$, where the effect of molecular collisions turns significant, and the transition from the nearly dipole to the monopole scattering pattern discussed in Sec. VI A should become visible.

Figure 6 illustrates the effect of gas rarefaction on the acoustic pressure field in response to Gaussian wall excitation. Similarly to Fig. 2, pressure colormaps are presented in the collisionless 

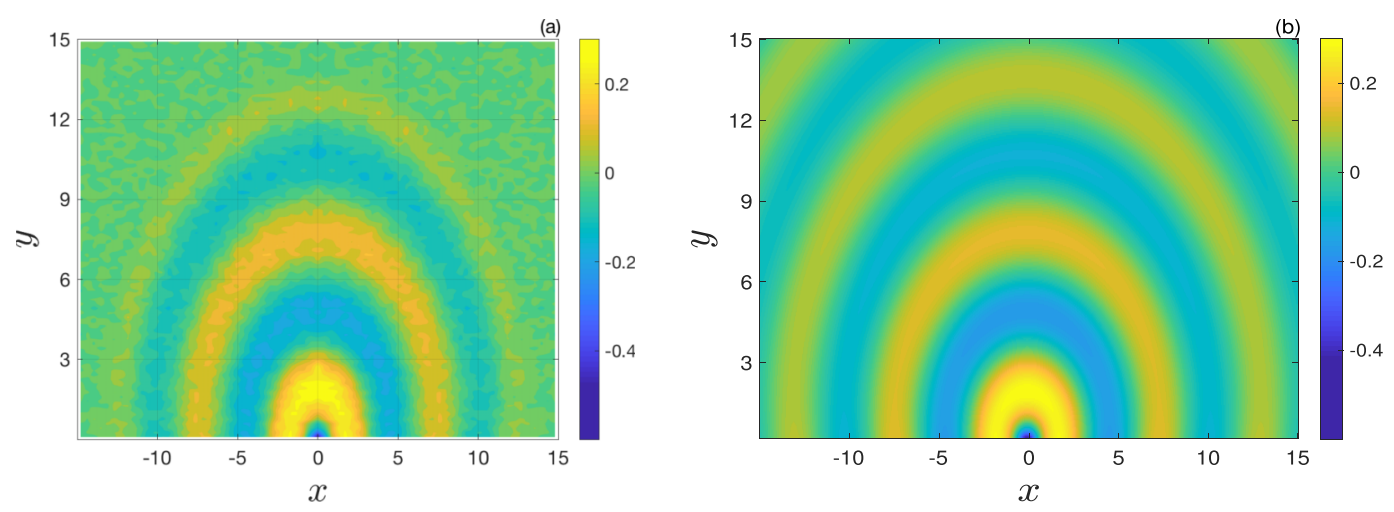

FIG. 7. Comparison between the DSMC (a) and slip-flow NSF (b) predicted pressure fields in response to Gaussian excitation with $\alpha=10$ and $\mathrm{Kn}=0.01$. The results are presented at quarter period, $t=\pi / 2$.

$[\mathrm{Kn} \rightarrow \infty$, Fig. 6(a)] and ideal-flow [Kn $\rightarrow$ 0, Fig. 6(f)] limits at quarter period time. Additionally, DSMC-calculated fields are shown in Figs. 6(b)-6(e) at the intermediate Knudsen numbers $\mathrm{Kn}=$ $5,0.5,0.1$, and 0.01 . The free-molecular and continuum limit results appear generally similar to their counterparts in Figs. 2(a) and 2(b), respectively, as both delta-function and Gaussian sources are confined to the vicinity of the origin and propagate symmetrically about the $y$ axis of wall motion.

Remarkably, the results in Fig. 6 demonstrate the transition between the nearly dipole $[\mathrm{Kn} \rightarrow \infty$, Fig. 6(a)] and monopole [Kn $\rightarrow 0$, Fig. 6(f)] acoustic patterns with decreasing rarefaction rate. Thus, at $\mathrm{Kn}=0.5$ [Fig. 6(c)], relatively weak "wavy" pressure oscillations are observed near the origin. With decreasing $\mathrm{Kn}$, the amplitude of these oscillations increases, as shown by the results in Figs. 6(d) and 6(e). At $\mathrm{Kn}=0.01$ (and, to some extent, also at $\mathrm{Kn}=0.1$ ), the decay rate of pressure oscillations becomes small enough so that the unwarranted effect of wave reflection at the virtual boundaries (placed at $x= \pm 15$ and $y=15$ ) is non-negligible. As explained in Sec. V, while this could be overcome by considering a larger domain size for the simulation, it would make the computation at small Knudsen numbers prohibitively time consuming, and was therefore not carried out here. The expected convergence of the $\mathrm{Kn} \ll 1$ result to its ideal-flow limit in Fig. 6(f) looks nevertheless apparent.

To better quantitate the effect of the virtual boundaries on DSMC results at small Kn, Fig. 7 compares between the DSMC-calculated pressure in Fig. 6(e) [repeated here as Fig. 7(a) for easy reference] and its counterpart NSF-calculated field obtained in Sec. IV B. While the two fields appear similar in the vicinity of source, the effect of the virtual boundaries, placed in the DSMC computation at $x= \pm 15$ and $y=15$, and missing in the NSF half-space calculation, is clearly visible through a "cutoff" vanishing of the acoustic field in Fig. 7(a) near the side and top surfaces. Recalling the characteristic inverse-square-root decay of the signal at continuum-limit conditions [see Eq. (42)], it is expected that the acoustic pressure fluctuation is yet significant at $O\left(10^{1}\right)$ distances from the source, turning its accompanying unwarranted effect of reflection by the virtual walls visible in the DSMC-calculated field.

\section{CONCLUSION}

We investigated the effect of gas rarefaction on the propagation of vibroacoustic sound in a half-space setup of a gas confined by a planar oscillating wall. Focusing on small-amplitude harmonic excitations imposed to only part of the surface, the problem was analyzed in the entire range of gas rarefaction rates, governed by the ratio between the gas mean free path and wavelength of a prescribed wall signal. Analytical solutions were derived in the freemolecular and continuum limits, consisting of the gas response to delta-function and Gaussian wall 
actuations. The analysis was supplemented, in the latter case, by direct simulation Monte Carlo calculations in the intermediate range of gas rarefaction rates. Remarkably, distinct differences were observed between sound propagation characteristics in the two limits. At continuum-limit conditions, the acoustic signal propagates isotropically in a monopole-type pattern, and decays inversely to the square root distance from the source. In contrast, the signal in the collisionless flow regime is exponentially decaying away from the source and follows a nearly dipole-type directivity field, with the acoustic pressure vanishing in the normal direction to the axis of source motion. The differences in results, stemming from the fundamentally distinct continuum and ballistic flow models, were discussed and rationalized in terms of the far-field limit-case descriptions.

Having considered the two-dimensional vibroacoustic configuration, several extensions may be carried out. To start with, the two-dimensional thermoacoustic problem of the pressure fluctuation generated by a locally thermally perturbed boundary may be readily studied. Here, boundary excitations may be imposed through either prescribed wall temperature variations or normal heat-flux nonuniformities. The combination of thermally and vibroacoustic-induced disturbances may then be considered to examine the feasibility of a "silent" vibrating wall, as was done in previous one-dimensional investigations [20-22]. Further extensions may include examination of confined setups, such as wall-bounded channels, where the effect of gas rarefaction on sound radiation by a point wall source can be studied. These constitute topics for future investigations.

\section{ACKNOWLEDGMENTS}

The research was supported by the Israel Science Foundation (Grant No. 1084/16) and the Aeronautical Engineering Research Fund.

\section{APPENDIX A: FAR-FIELD APPROXIMATIONS FOR THE SYSTEM RESPONSE TO A DELTA-FUNCTION EXCITATION}

\section{Free-molecular limit}

Abramowitz [34] applied the method of steepest descent to derive the asymptotic estimate

$J_{n}(y)=\int_{0}^{\infty} s^{n} \exp \left[-s^{2}-z / s\right] d s=3^{-n / 2} \zeta^{n / 2} \sqrt{\frac{\pi}{3}}\left[1+\frac{a_{n}^{(1)}}{\zeta}+\frac{a_{n}^{(2)}}{\zeta^{2}}+O\left(\zeta^{-3}\right)\right], \quad n=0,1, \ldots$,

where $z \gg 1, \zeta=3(z / 2)^{2 / 3}, \quad a_{n}^{(1)}=\left(3 n^{2}+3 n-1\right) / 12$, and $a_{n}^{(2)}=9 n^{4}+6 n^{3}-51 n^{2}-24 n+$ 25)/288. Setting $z=i y$ with $y \gg 1$, the quadratures $I_{n}$ in Eq. (38) may be expressed as

$$
I_{n}(y)=\sqrt{\pi} J_{n}(y)+2 J_{n+1}(y) .
$$

Subsequently, the far-field leading-order expressions for the free-molecular hydrodynamic perturbations in Eq. (36) are given by the real parts of

$$
\begin{aligned}
& \rho_{\mathrm{FM}}^{(\delta)}(t, x \sim O(1), y \gg 1)=\frac{2 \zeta^{2 / 3}}{y \sqrt{3 \pi}} \exp \left[i t-3 \zeta^{2 / 3}\right]\left[1+O\left(y^{-1 / 3}\right)\right], \\
& u_{\mathrm{FM}}^{(\delta)}(t, x \sim O(1), y \gg 1)=\frac{2 x \zeta}{y^{2} \sqrt{3 \pi}} \exp \left[i t-3 \zeta^{2 / 3}\right]\left[1+O\left(y^{-1 / 3}\right)\right], \\
& v_{\mathrm{FM}}^{(\delta)}(t, x \sim O(1), y \gg 1)=\frac{2 \zeta}{y \sqrt{3 \pi}} \exp \left[i t-3 \zeta^{2 / 3}\right]\left[1+O\left(y^{-1 / 3}\right)\right], \\
& p_{\mathrm{FM}}^{(\delta)}(t, x \sim O(1), y \gg 1)=\frac{4 \zeta^{4 / 3}}{3 y \sqrt{3 \pi}} \exp \left[i t-3 \zeta^{2 / 3}\right]\left[1+O\left(y^{-1 / 3}\right)\right] .
\end{aligned}
$$


The $\zeta^{-1}$ and $\zeta^{-2}$ corrections appearing in Eq. (A1) and omitted in Eq. (A2) for brevity become nonsmall with decreasing distance $y$ from the wall. Substituting $\zeta=3(i y / 2)^{2 / 3}$ in the expression for $p_{\mathrm{FM}}^{(\delta)}$, we obtain the leading-order approximation stated in Eq. (39).

\section{Continuum limit}

Inspecting Eq. (41) and rescaling

$$
\tilde{x}=x \sqrt{6 / 5} \text { and } \tilde{y}=y \sqrt{6 / 5},
$$

we seek to estimate

$$
I(\tilde{x}, \tilde{y})=\int_{-\infty}^{\infty} F(s) \exp \left[i\left(s \tilde{x}+\sqrt{1-s^{2}} \tilde{y}\right)\right] d s
$$

for $\tilde{y} \gg 1$ and $\tilde{x} \sim O(1)$. Within the $(-\infty,-1]$ and $[1, \infty)$ intervals the integrand is exponentially small and their contributions are accordingly negligible. Yet, within $[-1,1]$ the exponential power is purely imaginary and we therefore focus on evaluating

$$
I(\tilde{x}, \tilde{y}) \approx \int_{-1}^{1} F(s) \exp \{i[s \tilde{x}+\phi(s) \tilde{y}]\} d s
$$

where

$$
\phi(s)=\sqrt{1-s^{2}}
$$

The integrand in Eq. (A4) has a stationary-phase point at $s_{0}=0$, where $\phi\left(s_{0}\right)=1$ and $\phi^{\prime \prime}\left(s_{0}\right)=-1$. Expanding about $s=s_{0}$, we find [35]

$$
I(\tilde{x} \sim O(1), \tilde{y} \gg 1) \approx \sqrt{\frac{2 \pi}{\tilde{y}}} F(0) \exp [i(\tilde{y}-\pi / 4)] .
$$

Using Eq. (A6), the far-field approximations for the $y$-velocity and pressure perturbations in Eq. (37) are

$$
v_{\mathrm{Eu}}^{(\delta)} \approx \sqrt{\frac{3}{5 \pi \tilde{y}}} \exp \left[i\left(t+\tilde{y}-\frac{\pi}{4}\right)\right] \text { and } p_{\mathrm{Eu}}^{(\delta)} \approx-\sqrt{\frac{2}{\pi \tilde{y}}} \exp \left[i\left(t+\tilde{y}-\frac{\pi}{4}\right)\right],
$$

valid for $\tilde{y} \gg 1$ and $\tilde{x} \sim O(1)$.

\section{APPENDIX B: FREE-MOLECULAR SYSTEM RESPONSE TO A GAUSSIAN EXCITATION}

We substitute $v_{w}^{(G)}(x)=\exp \left[-\alpha x^{2}\right]$ into the $\varepsilon$-scaled nondimensional form of Eq. (1) and then into Eqs. (9) and (10). Carrying out the $\xi_{x}$ quadratures, we obtain

$$
\begin{aligned}
& \rho_{\mathrm{FM}}^{(G)}(t, x, y)=\frac{1}{\sqrt{\pi}} \exp \left[i t-\alpha x^{2}\right] \int_{0}^{\infty} \frac{\sqrt{\pi}+2 \xi_{y}}{\sqrt{\xi_{y}^{2}+\alpha y^{2}}} \xi_{y} \exp \left[-i \frac{y}{\xi_{y}}-\xi_{y}^{2}+\frac{\alpha^{2} x^{2} y^{2}}{\xi_{y}^{2}+\alpha y^{2}}\right] d \xi_{y}, \\
& u_{\mathrm{FM}}^{(G)}(t, x, y)=\frac{\alpha x y}{\sqrt{\pi}} \exp \left[i t-\alpha x^{2}\right] \int_{0}^{\infty} \frac{\sqrt{\pi}+2 \xi_{y}}{\left(\xi_{y}^{2}+\alpha y^{2}\right)^{3 / 2}} \xi_{y}^{2} \exp \left[-i \frac{y}{\xi_{y}}-\xi_{y}^{2}+\frac{\alpha^{2} x^{2} y^{2}}{\xi_{y}^{2}+\alpha y^{2}}\right] d \xi_{y}, \\
& v_{\mathrm{FM}}^{(G)}(t, x, y)=\frac{1}{\sqrt{\pi}} \exp \left[i t-\alpha x^{2}\right] \int_{0}^{\infty} \frac{\sqrt{\pi}+2 \xi_{y}}{\sqrt{\xi_{y}^{2}+\alpha y^{2}}} \xi_{y}^{2} \exp \left[-i \frac{y}{\xi_{y}}-\xi_{y}^{2}+\frac{\alpha^{2} x^{2} y^{2}}{\xi_{y}^{2}+\alpha y^{2}}\right] d \xi_{y},
\end{aligned}
$$




$$
\begin{aligned}
p_{\mathrm{FM}}^{(G)}(t, x, y)= & \frac{1}{3 \sqrt{\pi}} \exp \left[i t-\alpha x^{2}\right] \int_{0}^{\infty}\left[\frac{\xi_{y}^{2}\left(\xi_{y}^{2}+\alpha y^{2}+2 \alpha^{2} x^{2} y^{2}\right)}{\left(\xi_{y}^{2}+\alpha y^{2}\right)^{2}}+2 \xi_{y}^{2}+1\right] \\
& \times \frac{\sqrt{\pi}+2 \xi_{y}}{\sqrt{\xi_{y}^{2}+\alpha y^{2}}} \xi_{y} \exp \left[-i \frac{y}{\xi_{y}}-\xi_{y}^{2}+\frac{\alpha^{2} x^{2} y^{2}}{\xi_{y}^{2}+\alpha y^{2}}\right] d \xi_{y},
\end{aligned}
$$

for the free-molecular density, $x$-velocity, $y$-velocity, and acoustic pressure perturbations, respectively, in response to a time-harmonic Gaussian wall source. The integrals are evaluated numerically and the real part in each expression is taken to obtain the physical solution.

[1] M. Greenspan, Propagation of sound in five monatomic gases, J. Acoust. Soc. Am. 28, 644 (1956).

[2] L. Sirovich and J. K. Thurber, Propagation of forced sound waves in rarefied gas dynamics, J. Acoust. Soc. Am. 37, 329 (1965).

[3] G. Maidanik, H. L. Fox, and M. Heckl, Propagation and reflection of sound in rarefied gases. I. Theoretical, Phys. Fluids 8, 259 (1965).

[4] J. Foch and G. E. Uhlenbeck, Propagation of Sound in Monatomic Gases, Phys. Rev. Lett. 19, 1025 (1967).

[5] S. K. Loyalka and T. C. Cheng, Sound-wave propagation in a rarefied gas, Phys. Fluids 22, 830 (1979).

[6] S. Stefanov, P. Gospodinov, and C. Cercignani, Monte Carlo simulation and Navier-Stokes finite difference calculation of unsteady-state rarefied gas flows, Phys. Fluids 10, 289 (1998).

[7] N. G. Hadjiconstantinou, Sound wave propagation in transition-regime micro- and nanochannels, Phys. Fluids 14, 802 (2002).

[8] D. Kalempa and F. Sharipov, Sound propagation through a rarefied gas confined between source and receptor at arbitrary Knudsen number and sound frequency, Phys. Fluids 21, 103601 (2009).

[9] H. Struchtrup, Resonance in rarefied gases, Contin. Mech. Thermodyn. 24, 361 (2012).

[10] J. Tamayo, Study of the noise of micromechanical oscillators under quality factor enhancement via driving force control, J. Appl. Phys. 97, 044903 (2005).

[11] I. Bargatin, I. Kozinsky, and M. L. Roukes, Efficient electrothermal actuation of multiple modes of highfrequency nanoelectromechanical resonators, Appl. Phys. Lett. 90, 093116 (2007).

[12] J. Iannacci, M. Huhn, C. Tschoban, and H. Pötter, RF-MEMS technology for future mobile and highfrequency applications: Reconfigurable 8-bit power attenuator tested up to $110 \mathrm{GHz}$, IEEE Electron Device Lett. 37, 1646 (2016).

[13] A. Manela, G. A. Radtke, and L. Pogorelyuk, On the damping effect of gas rarefaction on propagation of acoustic waves in a microchannel, Phys. Fluids 26, 032001 (2014).

[14] Y. Sone, Effect of sudden change of wall temperature in rarefied gas, J. Phys. Soc. Jpn. 20, 222 (1965).

[15] D. C. Wadsworth, D. A. Erwin, and E. P. Muntz, Transient motion of a confined rarefied gas due to wall heating or cooling, J. Fluid Mech. 248, 219 (1993).

[16] A. Manela and N. G. Hadjiconstantinou, On the motion induced in a gas confined in a small-scale gap due to instantaneous boundary heating, J. Fluid Mech. 593, 453 (2007).

[17] A. Manela and N. G. Hadjiconstantinou, Gas-flow animation by unsteady heating in a microchannel, Phys. Fluids 22, 062001 (2010).

[18] J. Nassios, Y. W. Yap, and J. E. Sader, Flow generated by oscillatory uniform heating of a rarefied gas in a channel, J. Fluid Mech. 800, 433 (2016).

[19] N. Z. Liu, D. R. Ladiges, J. Nassios, and J. E. Sader, Acoustic flows in a slightly rarefied gas, Phys. Rev. Fluids 5, 043401 (2020).

[20] A. Manela and L. Pogorelyuk, Cloaking via heating: Approach to acoustic cloaking of an actuated boundary in a rarefied gas, Phys. Fluids 26, 062003 (2014). 
[21] A. Manela and L. Pogorelyuk, Active noise control of a vibrating surface: Continuum and non-continuum investigations on vibroacoustic sound reduction by a secondary heat-flux source, J. Sound Vib. 358, 20 (2015).

[22] Y. Ben-Ami and A. Manela, Acoustic wave propagation at non-adiabatic conditions: The continuum limit of a thin acoustic layer, Phys. Rev. Fluids 5, 033401 (2020).

[23] D. Kalempa and F. Sharipov, Numerical modelling of thermoacoustic waves in a rarefied gas confined between coaxial cylinders, Vacuum 109, 326 (2014).

[24] Y. Ben Ami and A. Manela, Acoustic field of a pulsating cylinder in a rarefied gas: Thermoviscous and curvature effects, Phys. Rev. Fluids 2, 093401 (2017).

[25] Y. Ben-Ami and A. Manela, The sound of a pulsating sphere in a rarefied gas: Continuum breakdown at short length and time scales, J. Fluid Mech. 871, 668 (2019).

[26] L. Wu, Sound propagation through a rarefied gas in rectangular channels, Phys. Rev. E 94, 053110 (2016).

[27] Y. W. Yap and E. Sader, Sphere oscillating in a rarefied gas, J. Fluid Mech. 794, 109 (2016).

[28] M. N. Kogan, Rarefied Gas Dynamics (Plenum, New York, 1969).

[29] Y. Sone, Molecular Gas Dynamics: Theory, Techniques, and Applications (Birkhäuser, Boston, 2007).

[30] K. Aoki, C. Baranger, M. Hattori, S. Kosuge, G. Martalo, J. Mathiaud, and L. Mieussens, Slip boundary conditions for the compressible Navier-Stokes equations, J. Stat. Phys. 169, 744 (2017).

[31] G. Bird, Molecular Gas Dynamics and the Direct Simulation of Gas Flows (Clarendon, Oxford, 1994).

[32] T. M. M. Homolle and N. G. Hadjiconstantinou, Low-variance deviational simulation Monte Carlo, Phys. Fluids 19, 041701 (2007).

[33] D. R. Ladiges and J. E. Sader, Frequency-domain deviational Monte Carlo method for linear oscillatory gas flows, Phys. Fluids 27, 102002 (2015).

[34] M. Abramowitz, Evaluation of the integral $\int_{0}^{\infty} e^{-u^{2}-x / u} d u$, J. Math. Phys. 32, 188 (1953).

[35] C. M. Bender and S. A. Orszag, Advanced Mathematical Methods for Scientists and Engineers (Springer, New York, 1999). 